Trauma Berufskrankh 2012 · 14[Suppl 2]:164-170 DOI 10.1007/s10039-012-1862-0

Online publiziert: 14. April 2012

(c) Springer-Verlag 2012

M. Lautenbach ${ }^{1} \cdot A \cdot$ Zach $^{2} \cdot$ A. Eisenschenk $k^{2,3}$

${ }^{1}$ Abteilung obere Extremität, Handchirurgie und Mikrochirurgie, Immanuel Krankenhaus Berlin

${ }^{2}$ Zentrum für Handchirurgie, Ernst-Moritz-Arndt-Universität Greifswald

${ }^{3}$ Abteilung für Hand-, Replantations- und Mikrochirurgie, Unfallkrankenhaus Berlin

\title{
Winkelstabile Implantate an der Hand
}

steht dann der Anschein, dass winkelstabile Implantate immer besser als nichtwinkelstabile Implantate sind. Eine genauere Betrachtung dieser Implantate bei der Anwendung an Carpus, Metacarpus und den Phalangen ist aber notwendig.

\section{Nichtwinkelstabile Implantate}

Sie sind seit Jahren in der handchirurgischen Anwendung standardisiert. Die in der Regel erreichbaren Ergebnisse sind gut untersucht und umfangreich beschrieben. Vor allem die Wertigkeit der Nutzung im Bereich des Metacarpus ist weitgehend unstrittig. Hingegen steht die Frage nach der Anwendung von Platten im Bereich der Phalangen immer wieder aufgrund der häufigen Probleme mit resultierenden Bewegungseinschränkungen nach der Osteosynthese in der Diskussion [7, 16, 24].

Ist eine Fraktur der Ossa metacarpalia oder der Phalangen nicht durch kleinere Implantate (z. B. Kirschner-Drähte usw.) zu stabilisieren, liegen gleichseitige multipel-instabile Frakturen vor oder ist eine anatomische Rekonstruktion von knöchernen Strukturen nur durch eine überbrückende, Kraft tragende Osteosynthese zu erreichen, kommen heute oft nichtwinkelstabile Plattensysteme zur Anwendung. Durch diese können stabile Osteosynthesen erreicht werden, wenn dies durch Kirschner-Draht- oder Schraubenosteosynthesen nicht möglich ist [16].

Die wesentlichen Vorteile der modernen, nichtwinkelstabilen Platten sind dabei, weitgehend unabhängig vom Hersteller, besonders das flache Design, die verfügbare große Auswahl an Plattentypen, die meist einsetzbaren Doppellochreihenplatten, die dreidimensionalen Plattenformen usw. Bei den meisten Herstellern betragen die dünnste Plattenprofilhöhe um 0,6 $\mathrm{mm}$ und der kleinste Schraubendurchmesser um 1,2 mm. Werden größere Implantate benötigt, sind diese zur Auswahl meist (bei weitgehend allen Herstellern) auf den Osteosynthese-Racks schon in verschiedenen Stärken vorhanden (Plattenprofilhöhe dann meist um $1,0 \mathrm{~mm}$ und Schraubendurchmesser um $1,4 / 1,7 / 2,3 \mathrm{~mm})$. Der Werkstoff ist regelmäßig Titan [7, 24]. Gerade für Metacarpus und Phalangen ist die dünne Plattenprofilhöhe vorteilhaft, da schon anatomiebedingt wenig Platz vorhanden ist und weniger Weichteiladhärenzen bei schlanken Implantaten zu erwarten sind. Zugänge können bei geringerer Narbenbildung kleiner gehalten werden und oft ist bei guter erreichbarer Stabilität eine frühe aktive Nachbehandlung erreichbar, was gerade bei bikortikaler Verankerung von jeweils 3 Schrauben auf beiden Seiten einer Fraktur regelhaft der Fall ist [2, 6, 19, 24].

Nichtwinkelstabile Implantate erfordern generell eine feste Fixation der Platte am Knochen, um eine Stabilität zu erreichen. Sind die Schrauben dabei nicht fest im Knochen (im Regelfall bikortikal) $\mathrm{zu}$ verankern, ist ein Implantatversagen möglich. Besonders bei schwacher Knochenqualität, Trümmer- und Defektfrakturen kann dies problematisch sein und muss beachtet werden $[3,7,10,19]$. 
Tab. 1 Winkelstabile Implantate im

Immanuel Krankenhaus Berlin im Bereich

der Hand

\begin{tabular}{ll} 
Region & $\begin{array}{l}\text { Anzahl Patienten } \\
\text { (absolut) }\end{array}$ \\
\hline Carpus & 2 \\
\hline Os metacarpale I & 7 \\
\hline Os metacarpale II-V & 6 \\
\hline Phalangen & 0
\end{tabular}

\section{Winkelstabile Implantate}

Die technischen Voraussetzungen, damit eine Schraube winkelstabil in einer Platte verankert werden kann (Gewinde, Verblocken usw.), sind vielfach in der Literatur beschrieben $[1,19]$. Verschiedene Formen der Platten sind heute von den einzelnen Herstellern verfügbar. Multidirektionale Schraubenpositionierungen sind bei einigen Herstellern auch bei den kleinen winkelstabilen Implantaten möglich. Bei den meisten Herstellern liegen die geringste Plattenprofilhöhe um $0,8 \mathrm{~mm}$ und der kleinste Schraubendurchmesser um 1,5 mm. Größere Implantate sind auch bei den winkelstabilen Osteosynthese-Racks meist (bei weitgehend allen Herstellern) in verschiedenen Stärken vorhanden (Plattenprofilhöhe dann meist um 1,3/1,5 mm und Schraubendurchmesser um 2,0/2,3 mm). Der Werkstoff ist in der Regel Titan [4, 24].

Das winkelstabile Implantat bewirkt als Gesamteinheit aus Platte mit Schrauben die Stabilität. Es fungiert quasi als Fixateur interne. Eine monokortikale Schraubenverankerung, ohne Reduktion der erreichbaren Plattenstabilität, ist möglich, was z. B. im Bereich der Metakarpalknochen Vorteile in der Vermeidung denkbarer Beugesehnenläsionen bringen kann $[7,15,19]$.

Die erhöhte erreichbare Stabilität hat bei instabilen, mehrfragmentären Brüchen und Trümmer- sowie Defektfrakturen, ggf. pathologischen Frakturen, denkbare Vorteile. Theoretisch könnte eine geringere intraoperative Knochendarstellung mit besserem Erhalt der Knochenperfusion möglich sein, da die Platte nicht direkt, wie bei den nichtwinkelstabilen Implantaten, dem Knochen aufliegen muss [22]. Ob dies aber der handchirurgischen Realität entspricht, ist zu bezwei- feln. Allein schon die Frakturdarstellung, die Reposition und der anatomisch geringe vorhandene Weichteilraum erfordern oft, von diesem Gedanken abzuweichen.

Aufgrund der erhöhten Stabilität sollte eine frühe postoperative Mobilisierung in der Regel möglich sein [4, 24].

\section{Nichtwinkelstabile vs. winkelstabile} Implantate im Bereich der Hand

Aus den oben angeführten Daten bleibt festzustellen, dass - weitgehend herstellerunabhängig - winkelstabile Implantate für den Einsatz im Bereich der Hand in der Regel größere Schraubendurchmesser und höhere Plattenprofilhöhen aufweisen als nichtwinkelstabile Implantate. Dies gilt auch für die kleinsten und dünnsten heute verfügbaren Implantate. In der Regel ist auch die Plattenauswahl bei den winkelstabilen Implantaten geringer als bei den nichtwinkelstabilen $[4,5]$.

Natürlich ist in Zeiten knapper finanzieller Ressourcen im Gesundheitssystem auch der Implantatpreis zu beachten. Je nach Einkauf und Hersteller muss beim winkelstabilen Implantat für die Platte mit gleicher Form und Lochzahl ein um etwa $40 \%$ und für jede Schraube ein um etwa $42 \%$ höherer Preis im Vergleich zum nichtwinkelstabilen Implantat erwartet werden. Die Gesamtkosten des winkelstabilen Systems sind also deutlich höher $[4,5]$.

Kommt es beim winkelstabilen Implantat zum Versagen, liegt meist ein Problem des Gesamtsystems vor. Hingegen kann ein Versagen des nichtwinkelstabilen Implantats durch einzelne Schraubenlockerungen verursacht werden. Dies ist für winkelstabile Platten-SchraubenKombinationen nicht typisch $[19,24]$.

\section{Material und Methode}

Um eine Aussage treffen zu können, wie winkelstabilen Implantate bisher im eigenen Krankengut genutzt wurden, wurden im Rahmen einer retrospektiven Datenerfassung die im Zeitraum vom 01.01.200631.03.2011 im Immanuel Krankenhaus Berlin implantierten winkelstabilen Systeme erfasst. Diese wurden 4 Regionen der Hand zugeordnet ( $\bullet$ Tab. 1). Dabei wurde nicht unterschieden, ob eine winkelsta- bile Osteosynthese aufgrund einer Fraktur oder einer anderen Notwendigkeit erfolgte.

Insgesamt wurden bei 15 Patienten, 9 weiblich und 6 männlich, winkelstabile Platten mit Schrauben eingebracht. Bei 11 Patienten wurde die rechte, bei $4 \mathrm{~Pa}$ tienten die linke Hand operativ behandelt. Eine bekannte oder mittels Messverfahren diagnostizierte Osteoporose bestand bei keinem Patienten. Das Durchschnittsalter lag bei 42,5 Jahren.

Als Unfallereignis gaben 1 Patient einen Sportunfall, 2 Patienten einen Verkehrsunfall, 3 Patienten einen Arbeitsunfall und 7 Patienten einen Privatunfall an. Keine Zuordnung vornehmen konnten 2 Patienten.

Zusätzlich wurde anhand der vorhandenen Röntgenbilder vor und nach der Operation sowie der Operationsberichte und der Aktenlage bewertet, ob bei den erfassten Fällen auch andere Osteosyntheseformen ohne winkelstabile Implantate möglich gewesen wären.

Da keine retrospektive vergleichende Untersuchung zu den eingesetzten nichtwinkelstabilen Implantaten erfolgte, können die Ergebnisse auch nur deskriptiv betrachtet werden.

\section{Ergebnisse}

Bezogen auf jährlich etwa 3000 Operationen in der Abteilung obere Extremität, Handchirurgie und Mikrochirurgie im Immanuel Krankenhaus Berlin wurden insgesamt nur wenig winkelstabile Implantate im gesamten Zeitraum an der Hand eingesetzt.

\section{Carpus}

Im Bereich des Carpus wurde nur 2-mal eine winkelstabile Platte verwendet. Dies erfolgte einmal zum Erreichen einer mediokarpalen Teilarthrodese des Handgelenks bei SNAC III („scaphoid nonunion advanced collapse"). Regelhaft wird im Immanuel Krankenhaus Berlin bei der Durchführung der mediokarpalen Teilarthrodese eine Osteosynthese mit Kirschner-Drähten durchgeführt. Die Plattenosteosynthese hat hier im Behandlungskonzept keinen regelhaften Stellenwert. Die Zahl der winkelstabilen Platten im Carpusbereich ist daher auch sehr gering. 


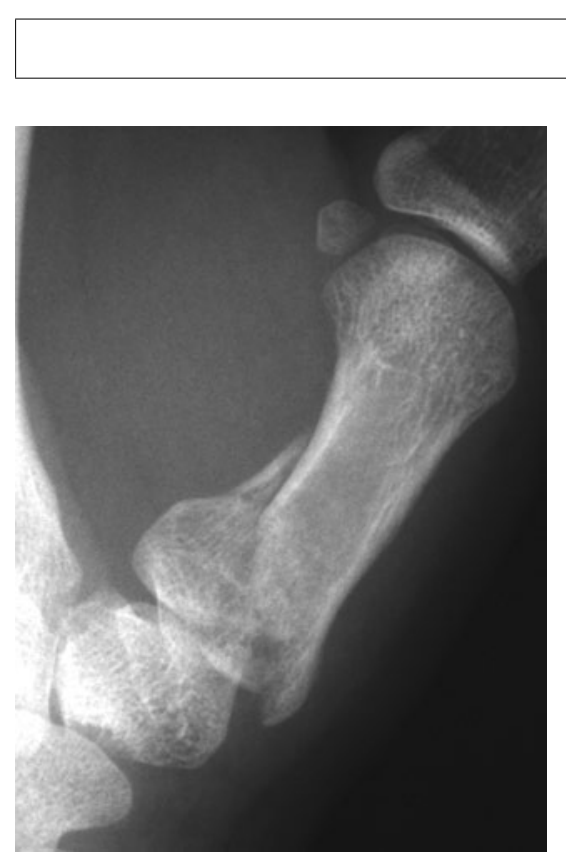

Abb. 1 \ Trümmerfraktur der Basis des Os metacarpale I

Im zweiten Fall erfolgte bei einer karpometakarpalen Luxationsfraktur mit Trümmerung der Gelenkfläche des CMC-V-Gelenks (CMC: karpometakarpal) eine Plattenosteosynthese des Os hamatum. Laut Operationsbericht wäre in diesem Fall ohne winkelstabiles Implantat nur schwer eine stabile Osteosynthese zu erreichen gewesen, da die Gelenkfläche zum Mittelhandknochen stark imprimiert und multifragmentär war. Die vorhandenen Röntgenbilder lassen keine weitergehende Bewertung zu.

\section{Mittelhand}

Im Bereich des ersten Mittelhandknochens wurden alle 7 winkelstabilen Implantate bei Basistrümmerfrakturen eingesetzt. Laut Operationsbericht und passend $\mathrm{zu}$ den vorhandenen Röntgenbildern wäre in diesen Fällen ohne winkelstabiles Implantat nur schwer eine stabile Osteosynthese zu erreichen gewesen. Im Bereich des Schafts oder des distalen ersten Mittelhandknochens erfolgte keine winkelstabile Plattenosteosynthese (『 Abb. 1, 2).

Im Bereich des 2. bis 5. Mittelhandknochens wurden bei 5 Patienten winkelstabile Implantate bei Basistrümmerfrakturen eingesetzt. Dies betraf bei einem Patienten das Os metacarpale IV, bei einem Patienten das Os metacarpale III (• Abb. 3), bei einem Patienten das Os metacarpale III-V als Mehrfachverletzung und bei

\section{Zusammenfassung $\cdot$ Abstract}

Trauma Berufskrankh 2012 - 14[Suppl 2]:164-170 DOI 10.1007/s10039-012-1862-0

C) Springer-Verlag 2012

\section{Lautenbach · A. Zach · A. Eisenschenk}

\section{Winkelstabile Implantate an der Hand}

\section{Zusammenfassung}

Zur Osteosynthese auch im Bereich der Hand stehen heute verschiedene Materialien zur Verfügung. Wie die Wertigkeit der winkelstabilen Implantate in dieser Lokalisation einzuschätzen ist, wird in der Literatur kontrovers diskutiert. Frakturen v. a. der Basen der Mittelhandknochen und besonders des ersten Metakarpalknochens stellen bei Trümmerung sicher gute Indikationen zur winkelstabilen Plattenosteosynthese dar. Aber nicht immer ist eine winkelstabile Versorgung im Bereich der Hand vorteilhaft. Die Nachteile des teuren und größeren Implantats müssen nicht in jedem Fall akzeptiert werden. Der überwiegende Anteil der Knochenrekonstruktionen an der Hand kann, wenn eine Operationsindi-

kation besteht, auch ohne winkelstabile Implantate gut chirurgisch versorgt werden, wobei nichtwinkelstabile Implantate, Schrauben- und Kirschner-Drahtosteosynthesen in Betracht kommen. Im Bereich der Phalangen sollten möglichst keine Platten-SchraubenOsteosynthesen erfolgen, da diese häufig mit schwachen funktionellen Ergebnissen einhergehen. Die diesbezügliche Entscheidung kann nur durch den behandelnden Handchirurgen getroffen werden.

\section{Schlüsselwörter}

Hand · Winkelstabile/nichtwinkelstabile Implantate - Osteosynthese - Metacarpus . Phalangen

\section{Locking implants at the hand}

\section{Abstract}

Currently there are various materials available for osteosynthesis in the region of the hand. The discussion in the literature about locking implants in the region of the hand is still continuing. Fractures of the bases of metacarpal bones and especially of the first metacarpals are good indications for locking plates but locking plates are not always advantageous. Most reconstructions of bones in the region of the hand can be achieved without using the thicker and more expensive implants. Non-locking implants, screws and Kirschner wires for osteosynthesis are mostly sufficient to achieve stable fixation but for phalangeal fractures plates should be avoided because of poor functional results. Only the hand surgeon can make the decision which of the various implants should be used.

\section{Keywords}

Hand - Locking/non-locking implants .

Osteosynthesis · Metacarpus .

Finger phalanges
2 Patienten das Os metacarpale V. Laut Operationsbericht und passend zu den vorhandenen Röntgenbildern wäre in diesen Fällen ohne winkelstabiles Implantat nur schwer eine stabile Osteosynthese zu erreichen gewesen.

Im Bereich des Schaftes oder der distalen Mittelhandknochen II-V erfolgte keine winkelstabile Plattenosteosynthese. Bei einem Patienten wurde zur Rekonstruktion des Os metacarpale IV bei vorhandener Pseudarthrose im distalen Knochenbereich nach Pseudarthrosenresektion und autologer Knochentransplantation eine winkelstabile Plattenosteosynthese durchgeführt. Da die distalen Schrauben sehr nah am Metakarpophalangealgelenk eingebracht werden mussten, wurde monokortikal-winkelstabil implantiert, um kei- ne palmare Schraubenlage ggf. im Grundgelenk zu verursachen. In der retrospektiven Analyse anhand der vorliegenden Röntgenbilder scheint eine Osteosynthese auch mit einer nichtwinkelstabilen Platte möglich gewesen zu sein.

\section{Phalangen}

In diesem Bereich wurde bei keinem Patienten eine Plattenosteosynthese mit einem winkelstabilen Implantat durchgeführt.

Regelhaft erfolgen im Immanuel Krankenhaus Berlin, wenn Osteosynthesen der Phalangen notwendig sind, diese mittels Kirschner-Drähten oder durch Schraubenosteosynthesen. Plattenosteosynthesen stellen hingegen eine absolute Aus- 


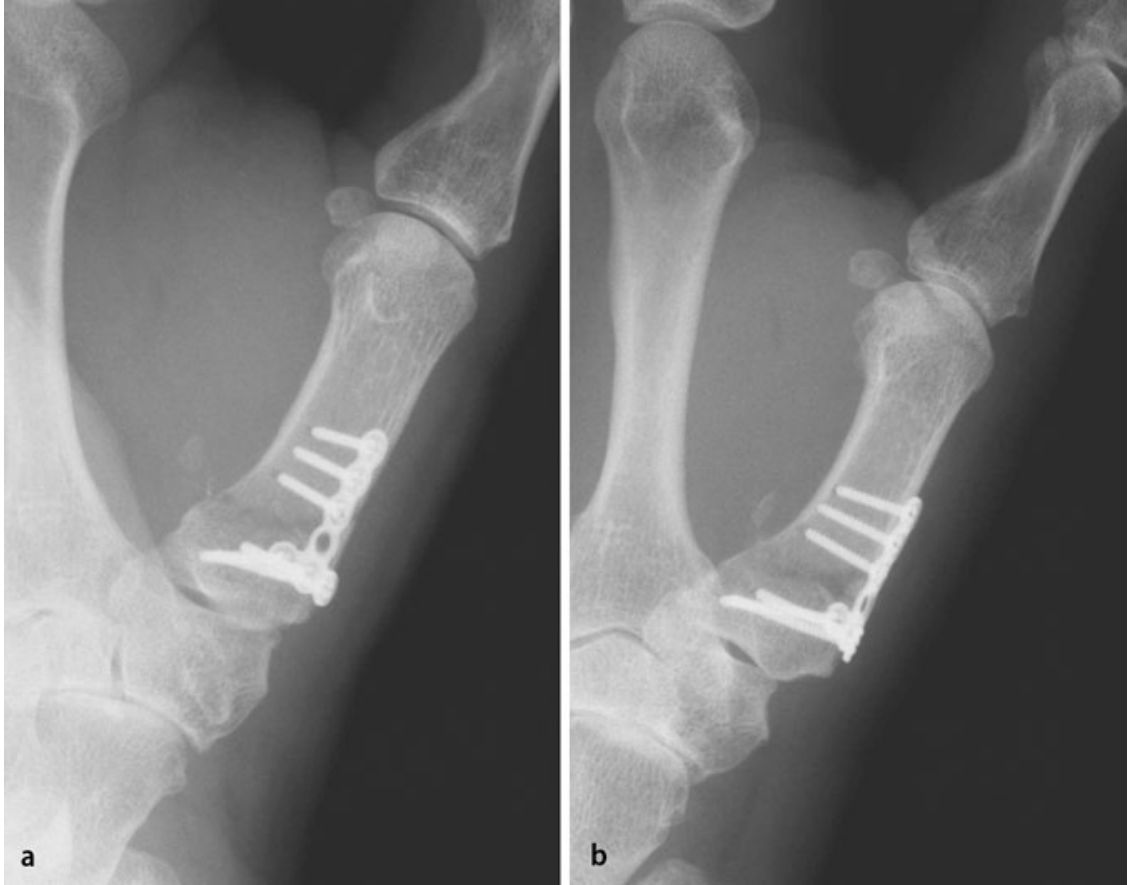

Abb. 2 ム Winkelstabile Plattenosteosynthese nach Trümmerfraktur der Basis des Os metacarpale I, a p.-a., b schräg
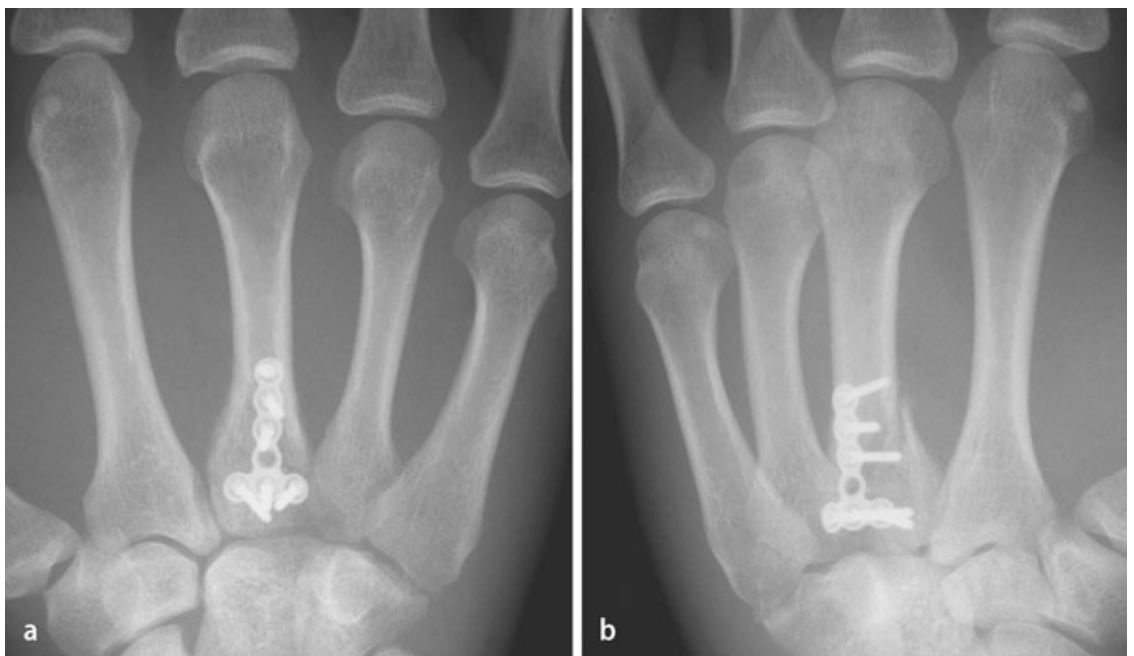

Abb. $3 \Delta$ \ Winkelstabile Plattenosteosynthese der Basis des Os metacarpale III, a p.-a., b schräg

nahme und Einzelfallentscheidung dar. Sie sind nicht regelhaft Bestandteil des Therapiekonzepts.

\section{Diskussion}

Winkelstabile Implantate für die Anwendung im Bereich der Hand sind auch in der kleinsten heute verfügbaren Größe, weitgehend herstellerunabhängig, teurer als nichtwinkelstabile Implantate und weisen größere Plattenprofilhöhen sowie einen größeren minimalen Schrau- der Hand, also am Carpus, Metacarpus und den Phalangen, ist notwendig [24].

\section{Carpus}

Frakturen im Bereich des Carpus werden in der Regel durch Osteosynthesen mittels Schrauben (z. B. Herbert-SchraubenOsteosynthese) oder Kirschner-Drähten behandelt [11, 12]. Plattenosteosynthesen sind nur extrem selten notwendig. Zur Rekonstruktion schmerzfreier karpaler Stabilität bei partiell erhaltener Handgelenkfunktion (z. B. mediokarpale Teilarthrodese) wurden spezielle Platten entwickelt. Ob diese aber wirklich im Vergleich zur Nutzung von Kirschner-Drähten vorteilhaft sind, ist in der Literatur nicht einheitlich bewertet [13]. Die eigenen Ergebnisse lassen hier keine Wertung zu, da im Immanuel Krankenhaus Berlin für diese Indikation nur Drähte genutzt werden. Trotzdem bestand in unserem Haus bisher keine Notwendigkeit und auch nicht der Wunsch, winkelstabile Implantate in das eigene Konzept der Behandlungen im Bereich des Carpus einzubeziehen.

Sicher sind aber weitere vergleichende Studien notwendig, um eine fundierte Beschreibung der Wertigkeit dieser Platten im Carpusbereich zu ermöglichen. Gerade bei den Teilarthrodesen wird dies schon lange kontrovers diskutiert [19].

\section{Metacarpus und Phalangen}

Im Bereich der oberen Extremität gehören die Frakturen des Metacarpus und der Phalangen zu den häufigsten Brüchen [3, 10, 24]. Nichtadäquat behandelte Frakturen können zu erheblichen Funktionsstörungen der Hand führen [22]. Das Ziel der Behandlung dieser Verletzungen muss die Wiederherstellung der Greiffunktion der Hand sein [5, 23]. Viele Brüche können dabei konservativ behandelt werden, wenn sie stabil zu redressieren sind und keine ausgeprägte Fehlstellung oder Gelenkverletzung bestehen.

Für die Entscheidung zwischen konservativer und operativer Therapie sind v. a.

- eine vorliegende Fehlstellung der Frakturfragmente,

- eine Achsenfehlstellung, 
- eine Rotationsfehlstellung der entsprechenden Finger,

- eine Verkürzung des entsprechenden Knochens,

- der Frakturtyp und

- der Patientenwunsch bzw. -bedarf

entscheidend [14]. Dislozierte Frakturen, intraartikuläre Frakturen mit Dislokation, mehrfragmentäre Frakturen usw. bedürfen aber häufig der operativen Therapie. Eine Vielzahl biomechanisch geeigneter Implantate steht hierfür zur Auswahl [10, 16, 22, 23]. Eine Besonderheit stellen die Rekonstruktionen bei Knochendefekten dar. Hier sind ggf. andere Überlegungen bei der Wahl der Osteosyntheseform nötig [14].

Basisfrakturen der Mittelhandknochen II-V erfordern bei Instabilität und Dislokation mit Luxationstendenz im Karpometakarpalgelenk die Osteosynthese. Hierfür kommen üblicherweise Drähte, Platten und Schrauben zur Anwendung. Gerade aber bei ausgeprägten Trümmerungen kann das Erreichen einer stabilen Reposition auch mit nichtwinkelstabilen Platten schwierig sein [18].

Die unter dem karpometakarpalen Knorpel gelegene knöcherne Substanz ist zusätzlich oft sehr weich. Hier können winkelstabile Platten eine subchondrale Abstützung bieten (etwas vergleichbar mit dem Einsatz der palmaren Implantate am distalen Radius), die Knochenlänge wiederherstellen und zusätzlich die ggf. vielen kleinen Fragmente stabil verankern. Das ist sicher sehr vorteilhaft und stellt eine gute Indikation für diese Implantate dar. Eine Transfixation mit Drähten zu Nachbarmetakarpalknochen kann dann entfallen. Im eigenen Haus wurden hierfür winkelstabile Implantate eingesetzt. Dies gilt auch für die Basisfraktur des 5. Mittelhandknochens, die durch den Zug der Sehne des M. extensor carpi ulnaris und der des M. adductor digiti minimi beeinflusst wird. Auch im Bereich der Basis des ersten Mittelhandknochens können gerade bei Trümmerfrakturen die winkelstabilen Platten eine stabile Frakturreposition und -retention ermöglichen, wenn dies mit nichtwinkelstabilen Implantaten nicht mehr erreicht werden kann. Wir sehen gerade hier eine gute Indikation für den Einsatz der winkelstabilen Implanta- te, was sich auch mit den Darstellungen in der Literatur deckt $[4,18]$.

Schaftfrakturen mit Angulationen der Fragmente von mehr als $30^{\circ}$ des 5., $20^{\circ}$ des 4. und $10^{\circ}$ des 3 . und 2. Metakarpalknochens werden reponiert. Schräg- und Spiralfrakturen neigen zur Verkürzung des entsprechenden Mittelhandknochens. Während Verkürzungen von $3 \mathrm{~mm}$ akzeptabel sind, müssen Rotationsfehler bei allen Mittelhandfrakturen beseitigt werden, um ein Überkreuzen der Finger nach Frakturheilung zu verhindern [14].

Subkapitale Metakarpalfrakturen zeigen häufig palmare Abkippungen des distalen Fragments. Während einige Autoren palmare Dislokationen der distalen 4 . und 5. Metacarpalia ab $20^{\circ}$ bzw. $30^{\circ}$ reponieren $[3,14]$, tolerieren andere Abkippungen bis $50^{\circ}$ oder $70^{\circ}[3,14]$. Am 2. und 3. Mittelhandkopf werden im Allgemeinen Angulationen von mehr als $10^{\circ}$ reponiert. Kopffrakturen des Mittelhandknochens mit Gelenkbeteiligung erfordern meist die operative Reposition und Fixation zur exakten Wiederherstellung der Gelenkfläche, um spätere Arthrosen zu vermeiden [5, 14].

Mittels Schraubenosteosynthesen und nichtwinkelstabiler Platten- und Schraubenosteosynthesen lassen sich im Bereich des Schafts von Mittelhandknochen stabile Frakturfixationen erzeugen. Eine frühe Mobilisierung der Hand ist somit, bei Gewährleistung der axialen und Rotationsstabilität, möglich. Eine winkelstabile Osteosynthese ist in der Regel nicht nötig [17]. Implantate mit größerer Plattenprofilhöhe sind dann eher nachteilig.

Mit der intramedullären Osteosynthese durch modellierte Kirschner-Drähte können in fast allen Fällen von distalen Metakarpalfrakturen (Kopf und subkapital) klinisch und radiologisch sehr gute Ergebnisse erzielt werden, bei sehr guter Patientenzufriedenheit. Ein Längenausgleich und eine vollständige Aufhebung der palmaren Abkippung des distalen Metakarpalknochens gelingt regelhaft. Es kommt zu keiner intraartikulären Implantatlage. Eine Nähe zum Streckapparat besteht durch den lateralen proximalen $\mathrm{Zu}$ gang nicht. Zusätzlich handelt es sich um eine schnelle (kurze Operationszeit) und preiswerte (kein spezielles Instrumentarium oder Implantat notwendig) Methode $[5,14]$. Bei Kopffrakturen mit Gelenkbe- teiligung kann ein anderes Vorgehen nötig sein. In keinem Fall war aber bei unseren Patienten die Anwendung eines winkelstabilen Implantats erforderlich.

Einige Autoren beschrieben, dass nur durch Platten und Schrauben eine stabile Osteosynthese bei Frakturen der distalen Mittelhandkochen erreicht werden kann $[3,5]$. Auch eine geringere Beeinträchtigung der Streckerhaube im Grundgelenkbereich durch winkelstabile Implantate ist beschrieben, da die Schraubenköpfe in der Platte versenkt werden [20, 21]. Hier wird aber vergessen, dass gerade die winkelstabilen Platten dicker sind und besonders vom Knochen abstehen, was die Streckerhaube in ihrer Funktion negativ beeinflussen muss. Zusätzlich kommt das Implantat auch nah an Gelenk, Band und Kapsel zu liegen, was die Grundgelenkfunktion erheblich beeinträchtigen kann. Dies deckt sich auch mit den in der Literatur beschriebenen Ergebnissen [5].

In der Literatur ist für die Therapie von Frakturen der Mittelhandknochen und Phalangen häufig die winkelstabile Plattenosteosynthese als besonders nützlich beschrieben, da sie aufgrund der hohen primären Stabilität eine sofortige Übungsstabilität erreichen lassen soll [20, 21]. Da aber gerade diese Implantate besonders vom Knochen vorstehen und zusätzlich eine größere Plattenprofilhöhe, mehr noch als bei den nichtwinkelstabilen Implantate bereits vorhanden, aufweisen, resultieren häufig Probleme mit dem Strecksehnengleitgewebe [5].

\section{Komplikationen}

Ihre Rate nach Plattenosteosynthesen im Bereich der Hand variiert in der Literatur stark, ihre Art ist hingegen meist ähnlich. Besonders nach Plattenosteosynthesen im Bereich der Phalangen sind Gelenkeinsteifungen, Strecksehnenadhärenzen und anders verursachte Bewegungseinschränkungen der Finger häufig. Ausbleibende Knochenheilungen im Bereich der Metacarpalia und der Phalangen nach Plattenosteosynthesen werden hingegen sehr selten berichtet [17]. 


\section{Indikationen/winkelstabil vs. nichtwinkelstabil}

Die in der Regel erreichbaren Ergebnisse nach Plattenosteosynthesen im Bereich der Hand sind gut untersucht und umfangreich beschrieben. Vor allem die Wertigkeit der Nutzung im Bereich des Metacarpus ist weitgehend unstrittig [14].

Hingegen steht die Frage nach der Anwendung von Platten im Bereich der Phalangen aufgrund der häufigen Probleme mit resultierenden Bewegungseinschränkungen nach Osteosynthese immer wieder in der Diskussion. Die Frakturbehandlung der Finger muss wesentlich deren komplexe funktionelle Anatomie berücksichtigen und eine größtmögliche Weichteilschonung besonders des Sehnengleitgewebes erreichen. Die funktionelle Wiederherstellung des Fingers muss das Hauptziel sein. Da die Phalangen nahezu vollständig mit Sehnen und Sehnengleitgewebe bedeckt sind, sollte nur bei speziellen Indikationen eine Plattenosteosynthese durchgeführt werden. Das Erzwingen einer größtmöglichen primären Stabilität ohne Berücksichtigung der Anatomie des Fingers muss zur Bewegungseinschränkung führen. Sind operative Stabilisierungen von Phalangenfrakturen notwendig, können meist durch das Einbringen von Kirschner-Drähten oder kleinstmöglichen Schrauben, unter Schonung der umgebenden Weichteile, für eine frühe Mobilisierung ausreichende Fixationen der Frakturfragmente erreicht werden. Dislozierte multifragmentäre Brüche der Mittelgliedbasis können z. B. durch dynamische Nachbehandlungen im Extensionsfixateur (z. B. SuzukiFixateur) unter dem Ziel eines Gelenkflächenremodellings behandelt werden. Auch hier besteht keine Indikation für ein winkelstabiles Implantat [23]. Seltene Indikationen für Plattenosteosynthesen sind nach Literaturdarstellung Defekt- und Trümmerfrakturen sowie ggf. veraltete Defektfrakturen am Grundglied [23]. Diese Darstellung deckt sich weitgehend auch mit dem eigenen Behandlungskonzept. Aber selbst bei Trümmerfrakturen muss besonders kritisch erwogen werden, ob wirklich eine Plattenosteosynthese nötig ist oder ob nicht Kirschner-Drähte die bessere Option darstellen. Bei einer winkelstabilen Plattenosteosynthese im Bereich der Phalangen sind aufgrund des größeren Implantatumfangs im Vergleich zur nichtwinkelstabilen Plattenosteosynthese noch mehr Probleme hinsichtlich der erreichbaren Funktionalität des Fingers zu erwarten.

Theoretisch sollte beim Implantieren winkelstabiler Platten eine geringere intraoperative Knochendarstellung mit besserem Erhalt der Knochenperfusion möglich sein, da die Platte nicht direkt, wie bei den nichtwinkelstabilen Implantaten, dem Knochen aufliegen muss [24]. Ob dies aber der handchirurgischen Realität entspricht, ist zu bezweifeln. Allein schon die Frakturdarstellung und -reposition sowie der anatomisch geringe Raum erfordern oft, von diesem Gedanken abzuweichen (s. oben). Ob dieser theoretische biomechanische und biologische Effekt überhaupt auch an der Hand zu einer besseren Knochenheilung führt, ist nicht durch veröffentlichte Studien belegt. Ungeklärt ist zudem, ob die zu rigide Stabilisierung eines winkelstabilen Implantats und die reduzierte interfragmentäre Kompression im Vergleich zum nichtwinkelstabilen Implantat im Bereich der Hand eine inadäquate Knochenheilung zur Folge haben kann. Während eine Vielzahl von Untersuchungen der Ergebnisse und von ausgebliebenen Knochenheilungen nach winkelstabilen Osteosynthesen an der unteren Extremität und proximal vom Radiokarpalgelenk veröffentlicht wurde, fehlen diese Daten für die Hand (Metacarpus und Phalangen). Auch eine vergleichende Arbeit der weiteren Komplikationen nach winkelstabiler und nichtwinkelstabiler Osteosynthese im Bereich der Hand wurde bisher nicht veröffentlicht [24].

\section{Materialentfernung}

Wie schon die Bezeichnung temporäre Implantate nahe legt, ist beim Einbringen von Platten und Schrauben am gesamten Bewegungsapparat zu bedenken, dass ggf. eine Materialentfernung notwendig sein kann. Im Bereich von Carpus, Metacarpus und Phalangen ist diese besonders bei der Nutzung von Titanimplantaten nicht regelhaft geplant und nach Literaturlage auch nicht regelhaft notwendig. Trotzdem kann z. B. bei störender Platte, Patienten- wunsch oder eingetretenen Bewegungseinschränkungen von Fingern eine Entfernung notwendig sein.

Nichtwinkelstabile Platten und Schrauben im Bereich der Hand zu entfernen, kann schwierig sein, und nicht immer gelingt dies bei fest im Knochen eingewachsenen Titanimplantaten komplett. Gerade Schrauben mit kleinem Durchmesser können schnell abbrechen und ggf. im Knochen verbleiben. Insbesondere winkelstabile Implantate im Bereich der Hand zu explantieren, kann ausgeprägt problematisch sein und das Risiko der Entstehung von Knochenläsionen beinhalten. Schon beim Einbringen muss daher auch dieses mögliche Problem bedacht werden $[8,14]$.

\section{Materialeigenschaften}

Die Vorteile von Titan als Werkstoff für Implantate im Bereich der Hand wurden vielfach beschrieben. Eine vergleichende Arbeit zu Stahlimplantaten im Bereich der Hand jedoch fehlt in der Literatur. Für die distalen Femurfrakturen sind nach Einbringen winkelstabiler Titanplatten vermehrte Kallusbildungen beschrieben [9]. Während diese in dieser Region ein gewünschter Effekt sind, können sie für die Fingerfunktion ein erhebliches Problem mit resultierender Bewegungseinschränkung darstellen, da schon kleine Umfangsvermehrungen des Knochens die Gleitfähigkeit und -amplitude von Sehnen beeinflussen können [2, 9, 24]. Dies gilt natürlich auch für alle nichtwinkelstabilen Implantate.

\section{Resümee}

Aus unserer Sicht sind sicher für die überwiegende Anzahl der Frakturen im Bereich der Metakarpalknochen nichtwinkelstabile Implantate, wenn eine Platten-Schrauben-Stabilisierung nötig ist, ausreichend und mit sehr guten Ergebnissen verbunden. Trotzdem sollte generell abgewogen werden, ob nicht andere Implantate (z. B. Kirschner-Drähte) zu bevorzugen sind, wie dies besonders im Bereich der distalen Mittelhandknochen meist auch erfolgt. Winkelstabile Implantate setzen wir nur bei Mehrfragmentfrakturen der Basen der Ossa metacarpalia (z. B. Metakarpale I bei Rolando-Frakturen, Trümmerfrakturen 
der Basen der Ossa metacarpalia II-V, Luxationsfrakturen mit Trümmerung der Basen) oder ggf. bei Defektfrakturen und deren Rekonstruktion ein. Ob auch bei osteoporotischem Knochen Vorteile bestehen, kann nur im Einzelfall beurteilt werden.

Bei Frakturen der Phalangen führen wird regelhaft keine Plattenosteosynthesen durch. Diese stellen in unserem Haus eine absolute Ausnahme dar und sind nicht üblicher Bestandteil unseres Behandlungskonzepts dieser Frakturen.

Stabilisierungen bei Frakturen der Handwurzelknochen (z. B. Skaphoid) und Knochenrekonstruktionen (z. B. Pseudarthrose) inklusive der Teilarthrodesen des Carpus führen wir mittels Schrauben- und Kirschner-Draht-Osteosynthesen durch. Die Notwendigkeit zur Anwendung von Platten besteht bei uns nur im seltenen Ausnahmefall. Ob dann wirklich ein winkelstabiles Implantat notwendig wird, ist fraglich.

Trotz der insgesamt wenig häufigen Anwendung von winkelstabilen Implantaten im Bereich der Hand in unserem Haus halten wir dauerhaft immer ein solches System vor. Gerade in den oben genannten Fällen (z. B. Basistrümmerfrakturen des ersten Mittelhandknochens) können intraoperative Situationen eintreten, die präoperativ nicht so umfangreich problematisch erwartet wurden. Hier ist mit den winkelstabilen Implantaten eine weitere Option zum Erreichen einer bestmöglichen operativen Versorgungsform gegeben, was auch ermöglicht werden sollte. Eine regelmäßige unkritische Anwendung unter der Vorstellung, dass winkelstabil einfach immer besser als nichtwinkelstabil ist, sollte unterbleiben.

Die weitere Entwicklung der winkelstabilen Implantate für die Anwendung an der Hand wird sicher auch deren Einsatzhäufigkeit im handchirurgischen Alltag beeinflussen. Sind kostengünstigere und kleinere Implantate verfügbar, wird sich ggf. auch deren Nutzung steigern. Trotzdem sollte immer gründlich abgewogen werden, wie viel Stabilität zum Erreichen einer optimalen Knochenheilung im Bereich der Hand tatsächlich notwendig ist. Die Entscheidung kann dabei nur durch den behandelnden Handchirurgen getroffen werden [23].

\section{Fazit für die Praxis}

Wie die Wertigkeit der winkelstabilen Implantate im Bereich der Hand einzuschätzen ist, wird in der Literatur kontrovers diskutiert. Frakturen v. a. der Basen der Mittelhandknochen und besonders des ersten Metakarpalknochens stellen bei Trümmerung sicher gute Indikationen dar. Aber nicht immer ist eine winkelstabile Versorgung im Bereich der Hand vorteilhaft. Die Nachteile des teuren und größeren Implantats müssen nicht immer hingenommen werden. Der überwiegende Anteil der Knochenrekonstruktionen im Bereich der Hand kann, wenn eine Operationsindikation besteht, auch ohne winkelstabile Implantate gut operativ therapiert werden. Im Bereich der Phalangen sollten, wenn immer möglich, keine Platten-Schrauben-Osteosynthesen erfolgen, da diese häufig mit schwachen funktionellen Ergebnissen verbunden sind. Die Entscheidung kann dabei nur durch den behandelnden Handchirurgen getroffen werden.

\section{Korrespondenzadresse}

Dr. M. Lautenbach

Abteilung obere Extremität, Handchirurgie und Mikrochirurgie,

Immanuel Krankenhaus Berlin,

Königstraße 63, 14109 Berlin

m.lautenbach@immanuel.de

Interessenkonflikt. Der korrespondierende Autor gibt für sich und seine Koautoren an, dass kein Interessenkonflikt besteht.

\section{Literatur}

1. Chou YC, Chen AC, Chen CY et al (2011) Dorsal and volar 2.4-mm titanium locking plate fixation for AO type $\mathrm{C} 3$ dorsally comminuted distal radius fractures. J Hand Surg [Am] 36:974-981

2. Cohen MS, Turner TM, Urban RM (2006) Effects of implant material and plate design on tendon function and morphology. Clin Orthop Relat Res 445:81-90

3. Day CS, Stern PJ (2011) Fractures of the metacarpals and phalanges. In: Wolfe SW, Hotchkiss RN, Pederson WC, Kozin SH (Hrsg) Green's operative hand surgery, 6. Aufl. Elsevier, Churchill Livingstone, Edinburgh London New York, S 240-282

4. Diaconu M, Facca S, Gouzou S, Liverneaux P (2011) Locking plates for fixation of extra-articular fractures of the first metacarpal base: a series of $15 \mathrm{ca}-$ ses. Chir Main 30:26-30
5. Facca S, Ramdhian R, Pelissier A et al (2010) Fifth metacarpal neck fracture fixation: locking plate versus K-wire. Orthop Traumatol Surg Res 96:506512

6. Freeland AE, OrbayJL (2006) Extraarticular hand fractures in adults: a review of new developments. Clin Orthop Relat Res 445:133-145

7. Gajendran VK, Szabo RM, Myo GK, Curtiss SB (2009) Biomechanical comparison of double-row locking plates versus single- and double-row nonlocking plates in a comminuted metacarpal fracture model. J Hand Surg [Am] 34:1851-1858

8. Gyuricza C, Carlson MG, Weiland AJ et al (2011) Removal of locked volar plates after distal radius fractures. J Hand Surg [Am] 36:982-985

9. Henderson CE, Kuhl L, Fitzpatrick DC, Marsh JL (2011) Locking plates for distal femur fractures: is there a problem with fracture healing? J Orthop Trauma 25:8-14

10. Henry MH (2008) Fractures of the proximal phalanx and metacarpals in the hand: preferred methods of stabilization. J Am Acad Orthop Surg 16:586-595

11. Herbert TJ, Fisher WE (1984) Management of the fractured scaphoid using a new bone screw. J Bone Joint Surg Br 66B:114-123

12. Herbert TJ, Fisher WE, Leicester AW (1992) The Herbert bone screw: a ten year perspective. J Hand Surg [Br] 17B:415-419

13. Krimmer H, Lanz U (2000) Post-traumatic carpal collapse. Follow-up and therapeutic concept. Unfallchirurg 103:260-266

14. Lautenbach M, Eisenschenk A (2000) Schaft- und Kopffrakturen der Mittelhandknochen. Trauma Berufskrankh [Suppl 3] 2:386-391

15. Ochman S, Doht S, Paletta J et al (2010) Comparison between locking and non-locking plates for fixation of metacarpal fractures in an animal model. J Hand Surg [Am] 35:597-603

16. Omokawa S, Fujitani R, Dohi Y et al (2008) Prospective outcomes of comminuted periarticular metacarpal and phalangeal fractures treated using a titanium plate system. J Hand Surg [Am] 33(6):857863

17. Page SM, Stern PJ (1998) Complications and range of motion following plate fixation of metacarpal and phalangeal fractures. J Hand Surg [Am] 23:827-832

18. Preisser $P$, Hülsbergen-Krüger $S$, Partecke $B D$ (2000) Proximale Metakarpalfrakturen mit Gelenkbeteiligung. Trauma Berufskrankh [Suppl 3] 2:379385

19. Ruchelsman DE, Mudgal CS, Jupiter JB (2010) The role of locking technology in the hand. Hand Clin 26:207-319

20. Towfigh $H$ (2011) Frakturen im Fingerbereich. In: Towfigh H, Hierner R, Langer M, Friedel R (Hrsg) Handchirurgie, Bd 1. Springer, Berlin Heidelberg New York, S 581-612

21. Towfigh $H$ (2011) Frakturen im Mitelhandbereich. In: Towfigh H, Hierner R, Langer M, Friedel R (Hrsg) Handchirurgie, Bd 1. Springer, Berlin Heidelberg New York, S 613-648

22. Windolf J, Rueger JM, Werber KD et al (2009) Behandlung von Mittelhandfrakturen. Unfallchirurg 112:577-589

23. Windolf J, Siebert $H$, Werber KD, Schädel-Höpfner M (2008) Behandlung von Fingerfrakturen. Unfallchirurg 111:331-339

24. Yaffe MA, Saucedo JM, Kalainov DM (2011) Nonlocked and locked plating technology for hand fractures. J Hand Surg [Am] 36:2052-2055 